\title{
Effective adsorbents for removal Cd(II) ion in solution from activated carbon of palm oil shell coated with magnetite particles
}

Buhani. ( $\sim$ buhani_s@yahoo.co.id)

University Lampung https://orcid.org/0000-0003-2289-1804

\section{Suharso .}

Universitas Lampung

Mita Rilyanti

Universitas Lampung

Miranda Sari

Universitas Lampung

Sumadi .

Universitas Lampung

\section{Research}

Keywords: Activated carbon, activated carbon-magnetite, adsorption, $\mathrm{Cd}(\mathrm{II})$ ion, palm oil shells

Posted Date: April 28th, 2020

DOl: https://doi.org/10.21203/rs.3.rs-23077/v1

License: (c) (1) This work is licensed under a Creative Commons Attribution 4.0 International License. Read Full License 


\section{Abstract}

Investigation of the ability of activated carbon (AC) adsorption from modification of palm oil shells by coating magnetite $\left(\mathrm{Fe}_{3} \mathrm{O}_{4}\right)$ particles to $\mathrm{Cd}(\mathrm{II})$ ions in solution was studied through a series of adsorption experiments with a bacth method. The activated carbon adsorbents (PPAC-P) and the activated carbonmagnetite (PPAC-MnPs) were characterized by Fourier transform spectrometer to identify the functional groups, X-ray diffraction to study the level of material crystallization, scanning electron microscopyenergy-dispersive X-ray analysis to analyze the surface morphology of material and element constituents, as well as Brunauer-Emmett-Teller analyzer to analyze the surface areas of the adsorbents. The concentrations of $\mathrm{Cd}(\mathrm{II})$ ions in the solution were determined using atomic absorption spectrophotometer. The adsorption process of $\mathrm{Cd}(\mathrm{II})$ ions by PPAC-P and PPAC-MnPs was optimum at pH 6 and 7 with contact time of 90 minutes, respectively. The adsorption kinetics of $\mathrm{Cd}(\mathrm{II})$ ions in PPAC-P and PPAC-MnPs have a tendency to follow the pseudo second-order kinetics model and the Freundlich adsorption isotherm model with a value of $R^{2}>0.99$. These suggested that the adsorption process of the $C d(I I)$ ions on PPAC-P and PPAC-PMnPs were described as heterogeneous adsorption. PPAC-PMnPs adsorbents can be used repeatedly with \% $\mathrm{Cd}(\mathrm{II})$ ions adsorbed almost constant for 4 cycles with adsorption efficiency > $80 \%$.

\section{Full Text}

This preprint is available for download as a PDF.

\section{Tables}

Table 1 Adsorption kinetic parameter for the adsorption of Cd(II) ion on PPAC-P and PPACMnPs.

\begin{tabular}{lccccc}
\hline \multirow{2}{*}{ Adsorbents } & \multicolumn{2}{c}{ Pseudo-first-order } & & \multicolumn{2}{c}{ Pseudo-second-order } \\
\cline { 2 - 3 } \cline { 5 - 6 } & $k_{1}\left(\mathrm{~min}^{-1}\right)$ & $R^{2}$ & & $\begin{array}{c}k_{2} \times 10^{-4} \\
\left(\mathrm{~g} \mathrm{mg}^{-1} \mathrm{~min}^{-1}\right)\end{array}$ & $R^{2}$ \\
\hline PPAC-P & 0.071 & 0.839 & & 8.240 & 0.982 \\
PPAC-PMnPs & 0.110 & 0.931 & & 40.640 & 0.993 \\
\hline
\end{tabular}

Table 2 Intra particle diffussion model for Cd(II) ion on PPAC-P and PPAC-MnPs

\begin{tabular}{lcccccc}
\hline Adsorbent & \multicolumn{2}{c}{ Initial linear portion } & \multicolumn{3}{c}{ Second linear portion } \\
\cline { 2 - 7 } & $\begin{array}{c}k_{\text {i1 }} \\
\left(\mathrm{mg} \mathrm{g}^{-1} \mathrm{~min}^{-0.5}\right)\end{array}$ & $\begin{array}{c}C_{1} \\
\left(\mathrm{mg} \mathrm{g}^{-1}\right)\end{array}$ & $R_{1}{ }^{2}$ & $\begin{array}{c}k_{i 2} \\
\left(\mathrm{mg} \mathrm{g}^{-1} \mathrm{~min}^{-0.5}\right)\end{array}$ & $\begin{array}{c}C_{2} \\
\left(\mathrm{mg} \mathrm{g}^{-1}\right)\end{array}$ & $R_{2}{ }^{2}$ \\
\hline PPAC-P & 4.316 & 9.414 & 0.975 & 1.716 & 29.554 & 0.821 \\
PPAC-PMnPs & 3.882 & 16.916 & 0.993 & 0.169 & 46.180 & 0.942 \\
\hline
\end{tabular}


Table 3 Langmuir and Freundlich parameters for the adsorption of $\mathrm{Cd}(\mathrm{II})$ ion on PPAC$\mathrm{P}$ and PPAC-MnPs.

\begin{tabular}{|c|c|c|c|}
\hline & Adsorbent & PPAC-P & PPAC-MnPs \\
\hline Models & Parameters & \multirow[b]{2}{*}{69.332} & \multirow[b]{2}{*}{80.400} \\
\hline \multirow{7}{*}{ Langmuir } & $q_{\text {exp }}\left(\mathrm{mg} \mathrm{g}^{-1}\right)$ & & \\
\hline & & & \\
\hline & $q_{m}\left(\mathrm{mg} \mathrm{g}^{-1}\right)$ & 92.131 & 104.074 \\
\hline & $K_{L} \times 10^{-4}\left(\mathrm{~L} \mathrm{mg}^{-1}\right)$ & 8.197 & 9.259 \\
\hline & $R^{2}$ & 0.852 & 0.819 \\
\hline & $R M S E$ & 3.599 & 4.363 \\
\hline & $\chi^{2}$ & 3.895 & 4.203 \\
\hline \multirow[t]{6}{*}{ Freundlich } & & & \\
\hline & $K_{F}\left(\mathrm{mg} \mathrm{g}^{-1}\right)\left(\mathrm{L} \mathrm{mg}^{-1}\right)^{1 / n}$ & 2.802 & 3.114 \\
\hline & $n$ & 1.430 & 1.382 \\
\hline & $R^{2}$ & 0.994 & 0.995 \\
\hline & $R M S E$ & 3.211 & 2.735 \\
\hline & $x^{2}$ & 1.232 & 0.896 \\
\hline
\end{tabular}

Table 4 The adsorption capacity of Cd(II) ion on PPAC-PMnPs compared with other adsorbents.

\begin{tabular}{lccc}
\hline \multicolumn{1}{c}{ Adsorbent } & $\begin{array}{c}q_{m}\left(\mathrm{mg} \mathrm{g}^{-}\right. \\
1)\end{array}$ & $\begin{array}{c}\text { Experimental } \\
\text { condition } \\
(\mathrm{pH})\end{array}$ & Reference \\
\hline Cd(II) ion-imprinted ionic polymer & 83.89 & 5.0 & {$[3]$} \\
Nannochloropsis sp.-Silica & 23.81 & 5.0 & {$[48]$} \\
\hline Urea-modified wheat straw & 39.22 & 6.0 & {$[49]$} \\
Olive stone activated carbon & 11.72 & 5.0 & {$[50]$} \\
Mercapto-modified graphene oxide & 36.0 & 5.0 & {$[51]$} \\
Carboxyl-modified jute fiber & 88.98 & 6.6 & {$[52]$} \\
Cellulose nanofiber & 45.90 & 4.0 & {$[53]$} \\
Iron-trimesic & 54.95 & 7.0 & This work \\
frameworks & & & \\
PPAC-PMnPs & 80.40 & 7.0 & \\
\hline
\end{tabular}

\section{Figures}




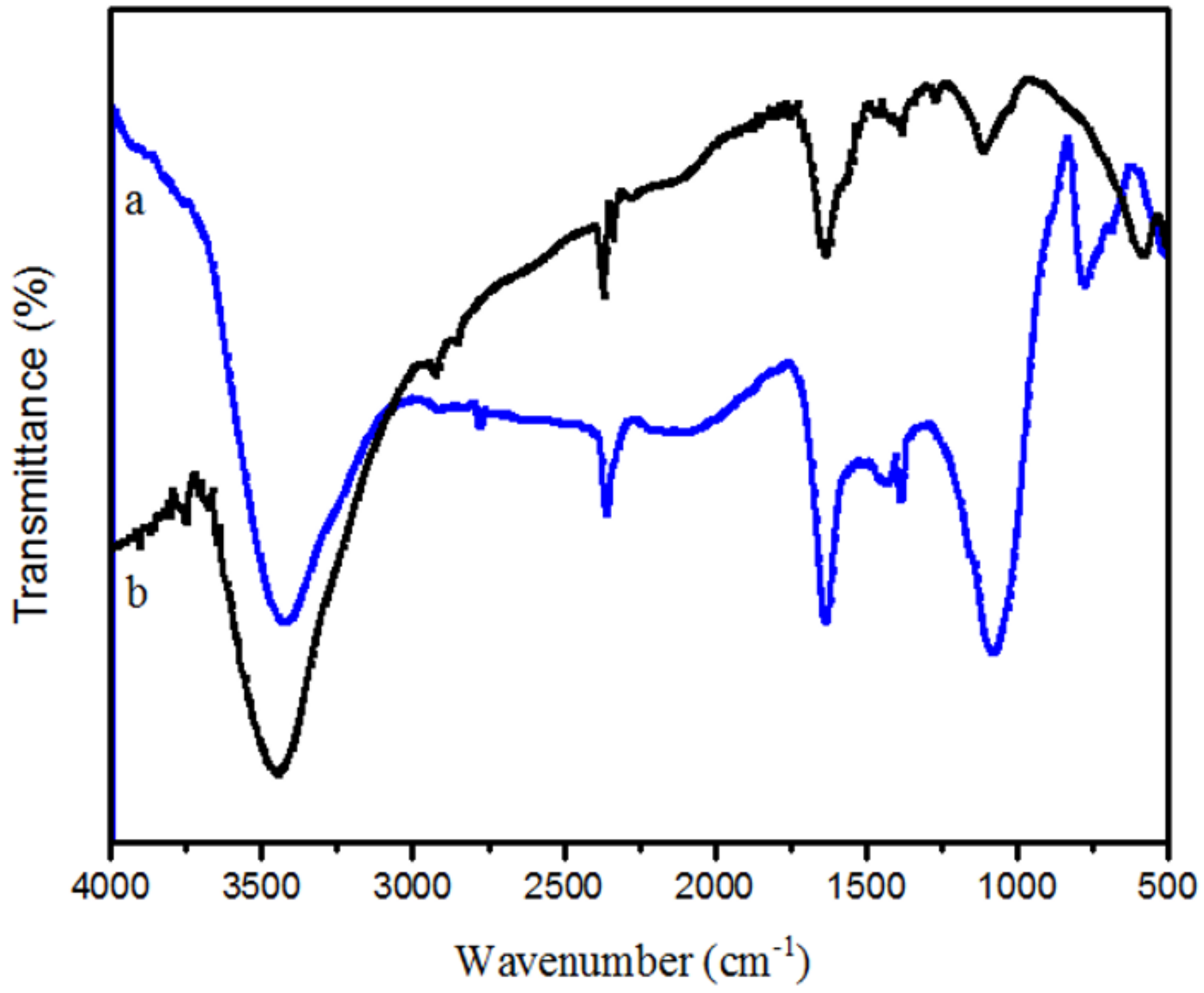

Figure 1

Infrared spectra of the (a) PPAC-P and (b) PPAC-PMnPs. 


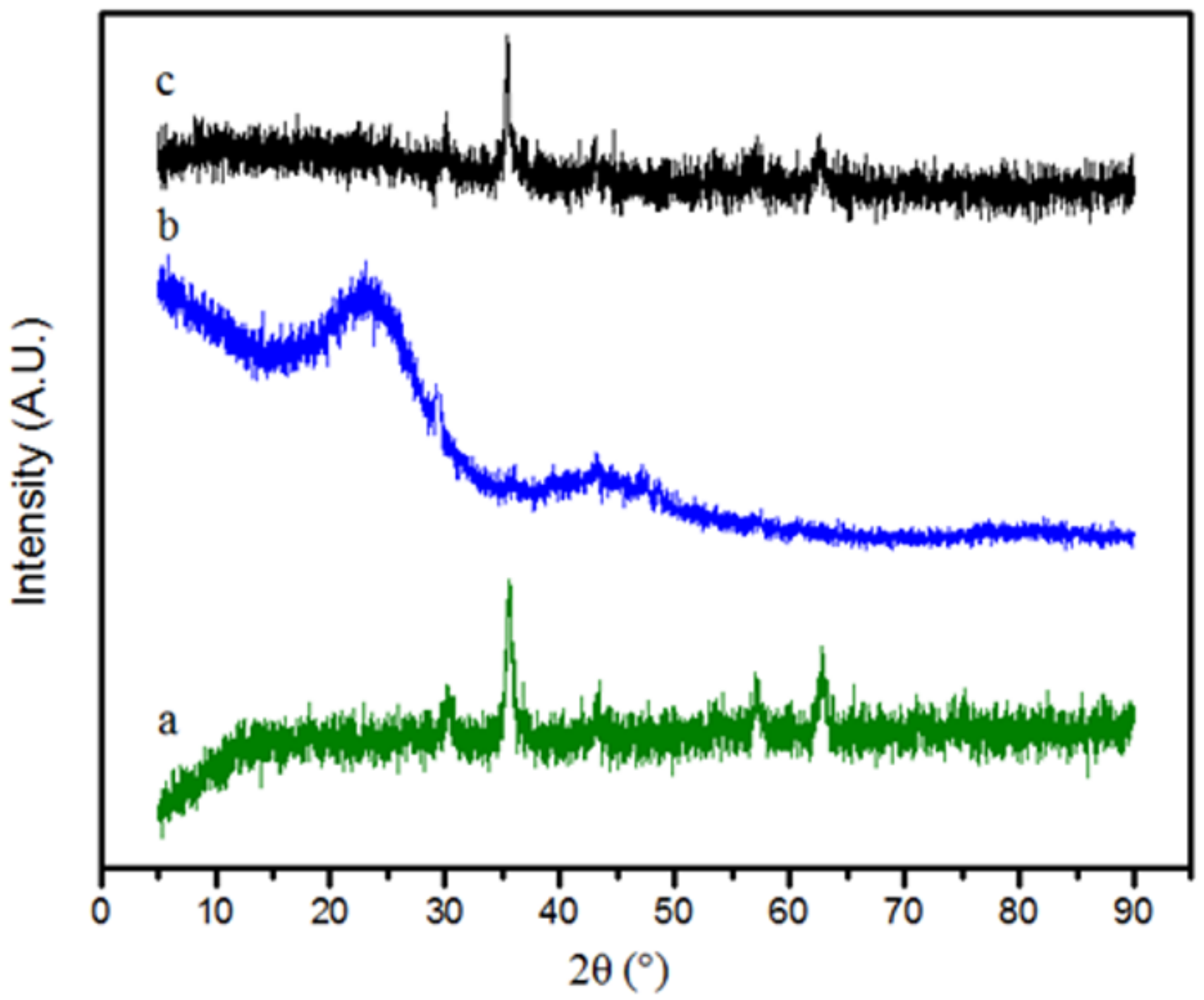

Figure 2

XRD patterns of (a) Magnetite, (b) PPAC-P, and (c) PPAC-MnPs. 

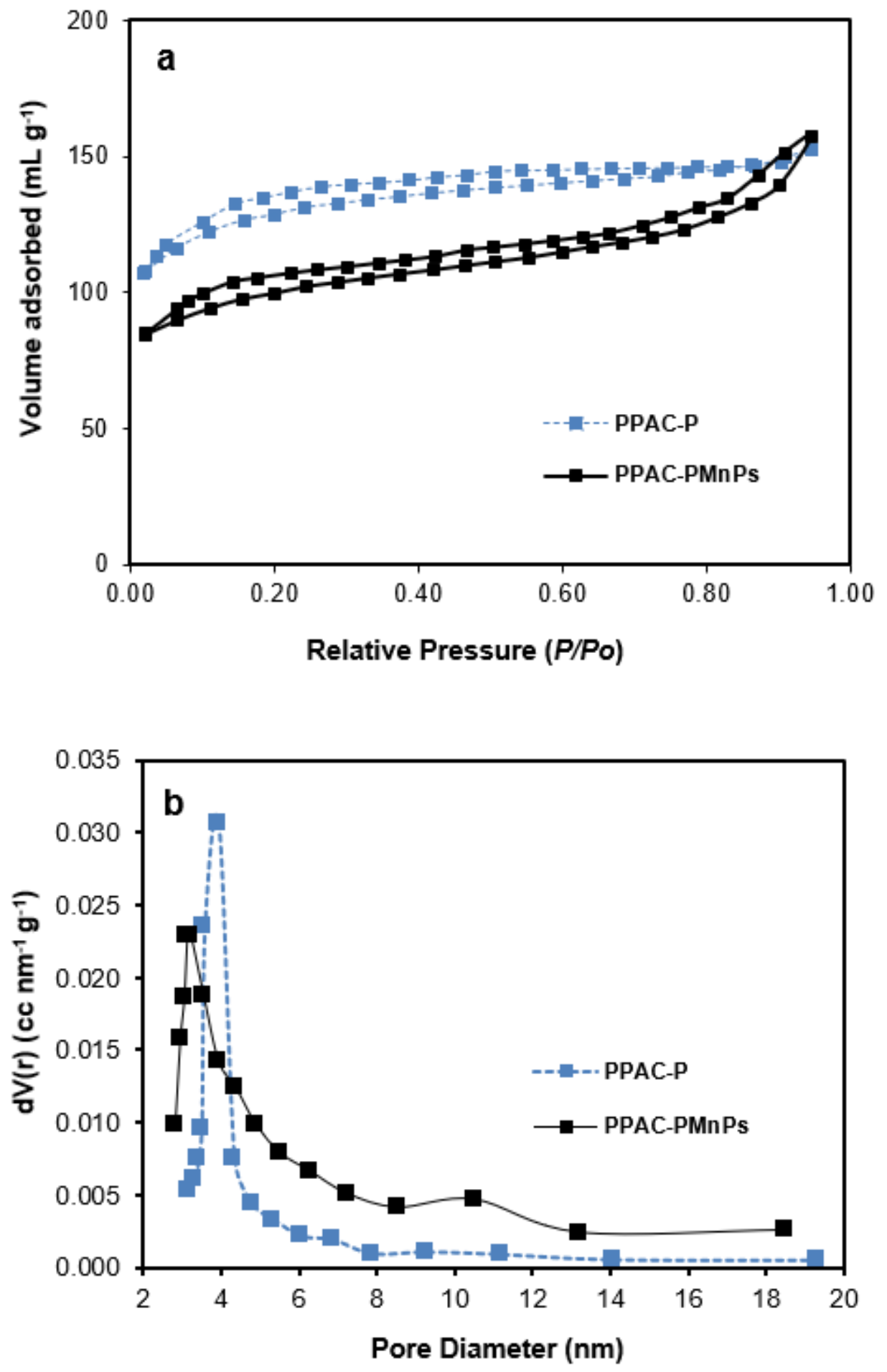

Figure 3

(a) N2 adsorption/desorption isotherms and (b) pore size distribution of PPAC-P and PPAC-PMnPs. 

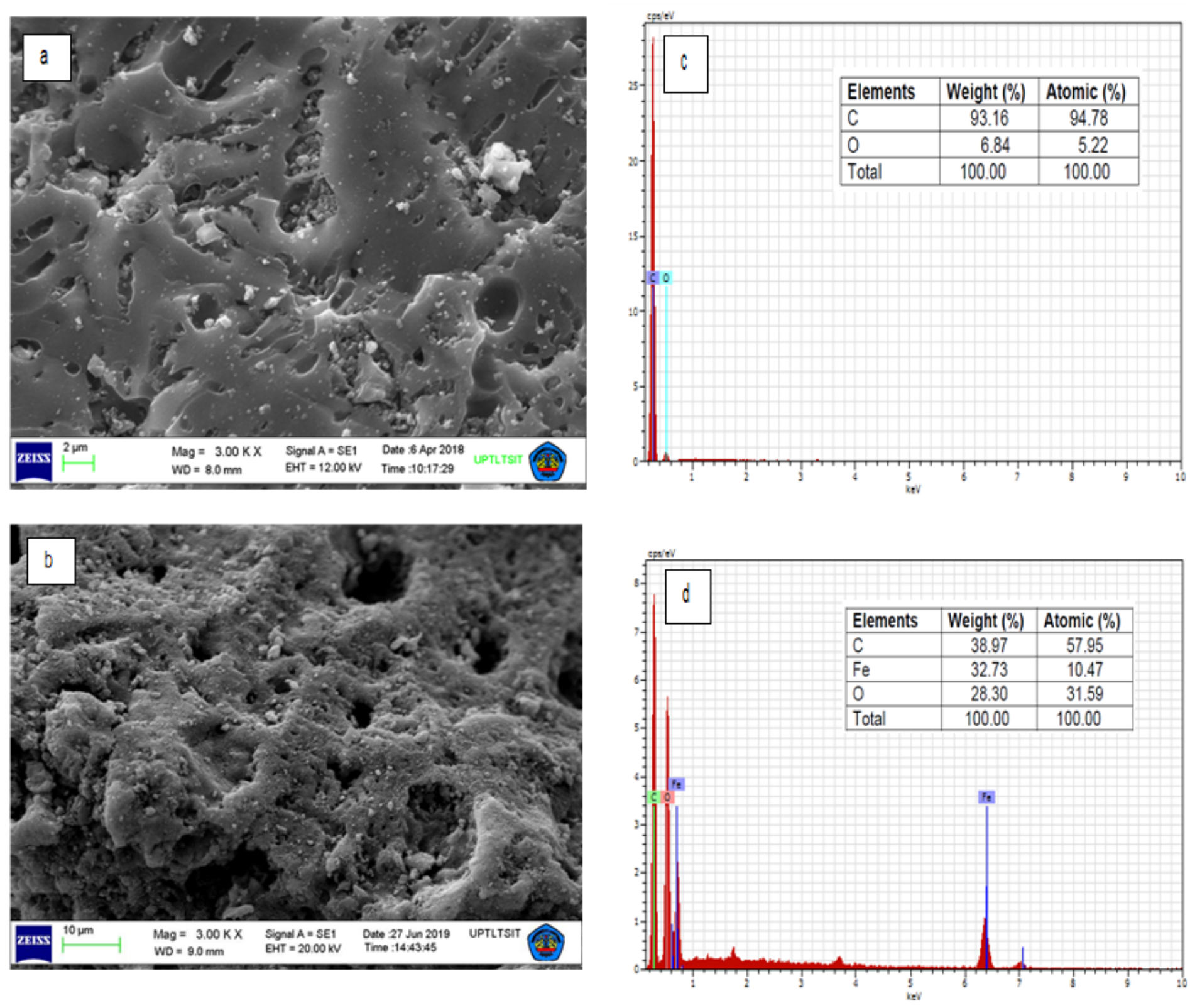

Figure 4

(a) N2 adsorption/desorption isotherms and (b) pore size distribution of PPAC-P and PPAC-PMnPs. 

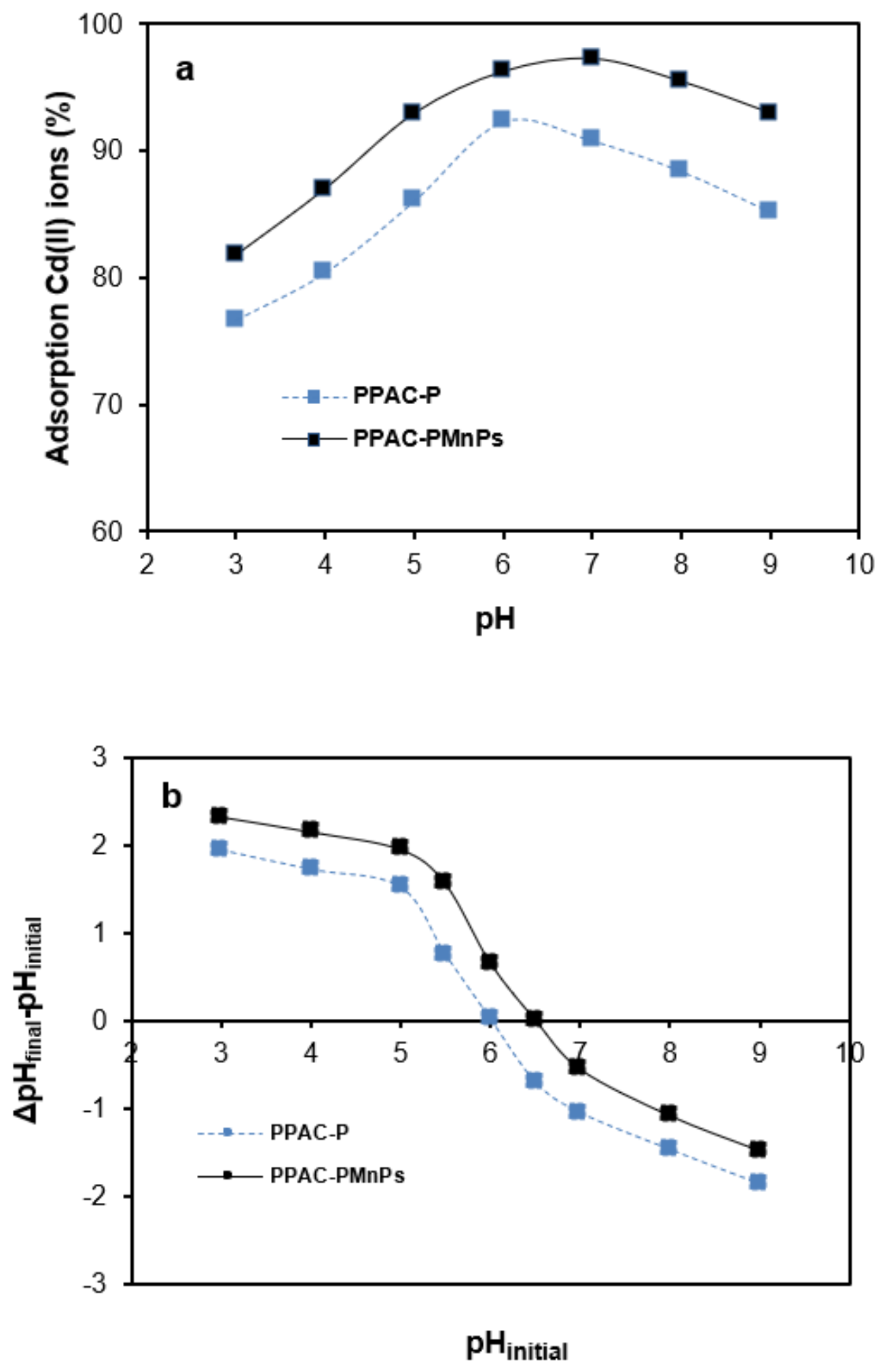

Figure 5

(a) N2 adsorption/desorption isotherms and (b) pore size distribution of PPAC-P and PPAC-PMnPs. 


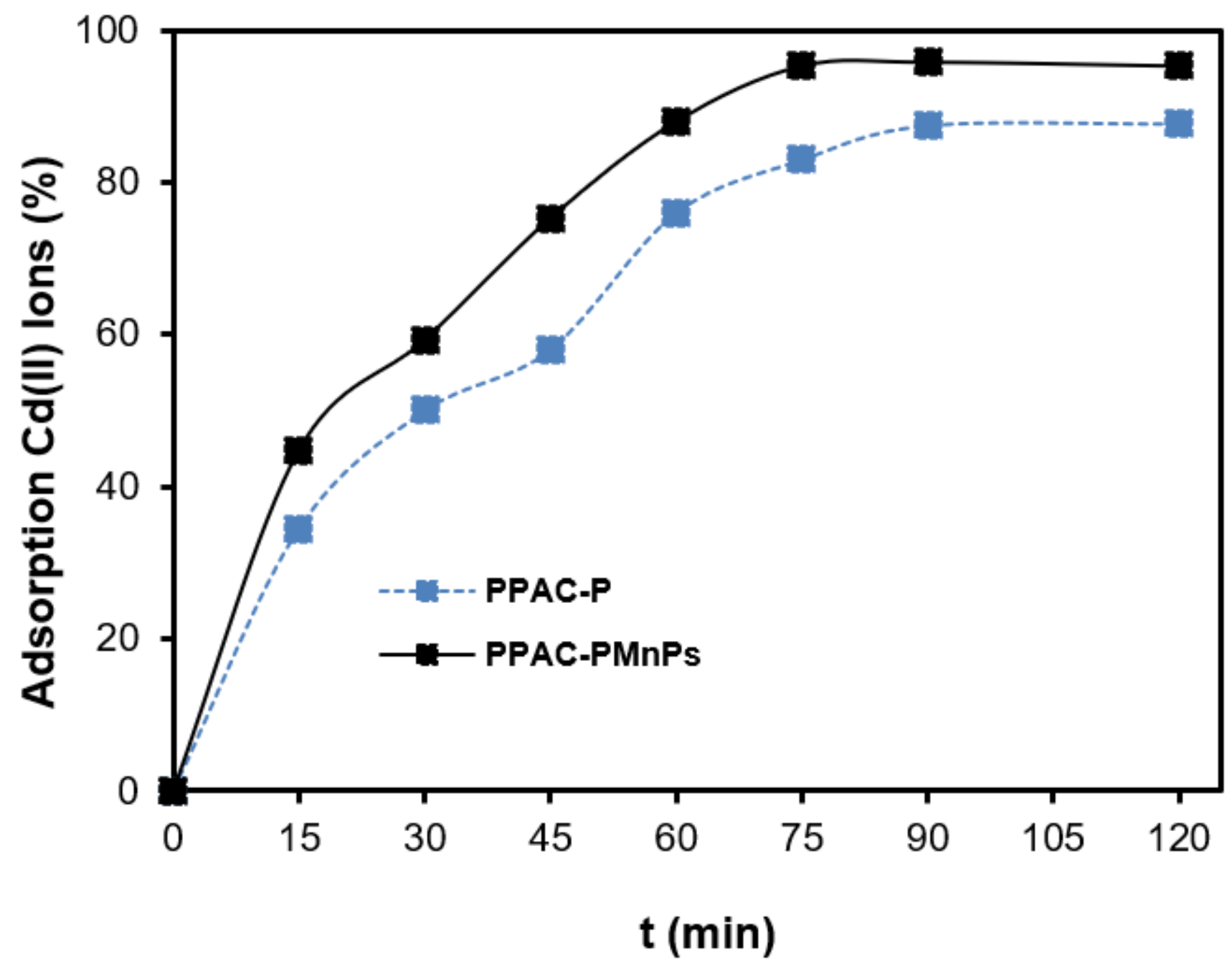

Figure 6

Influence of contact time on adsorption of Cd(II) ion onto PPAC-P and PPAC-PMnPs. 


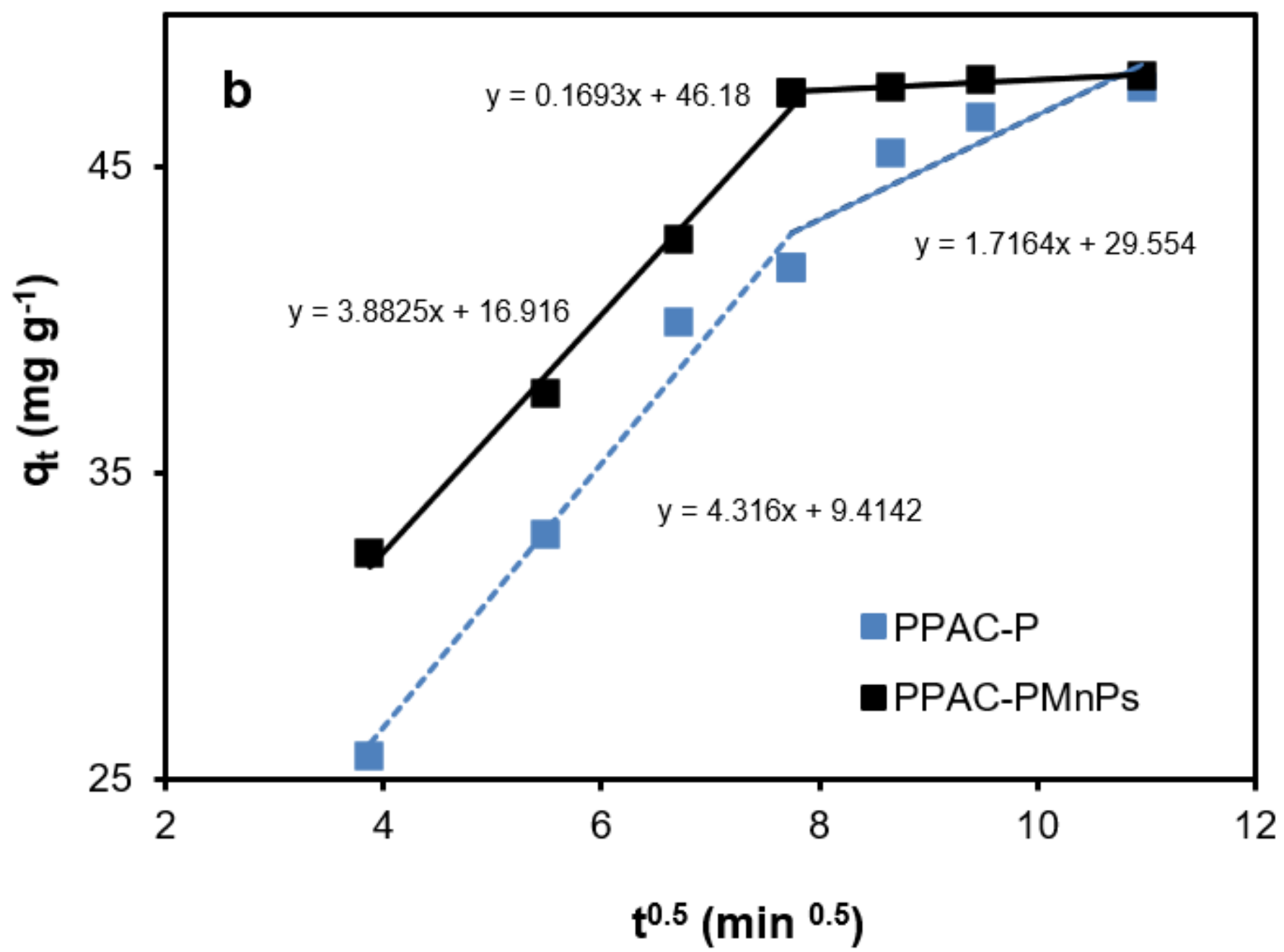

Figure 7

Intra-particle diffusion (IPD) model-predicted kinetics of $\mathrm{Cd}(\mathrm{II})$ ion sorption by PPAC-P and PPAC-MnPs. 

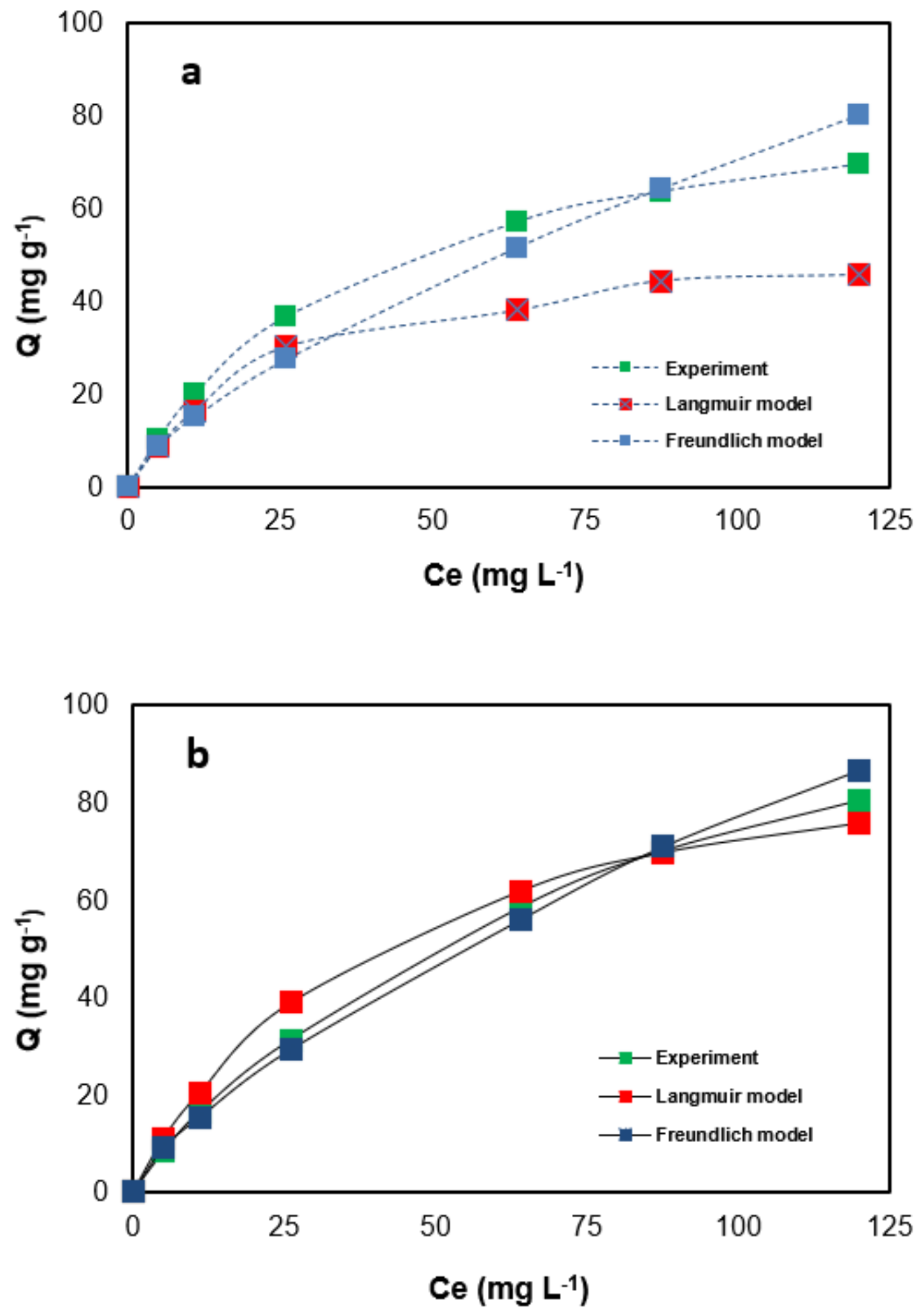

Figure 8

Adsorption isotherm model of Cd(II) ions on (a) PPAC-P dan (b) PPAC-MnPs. 


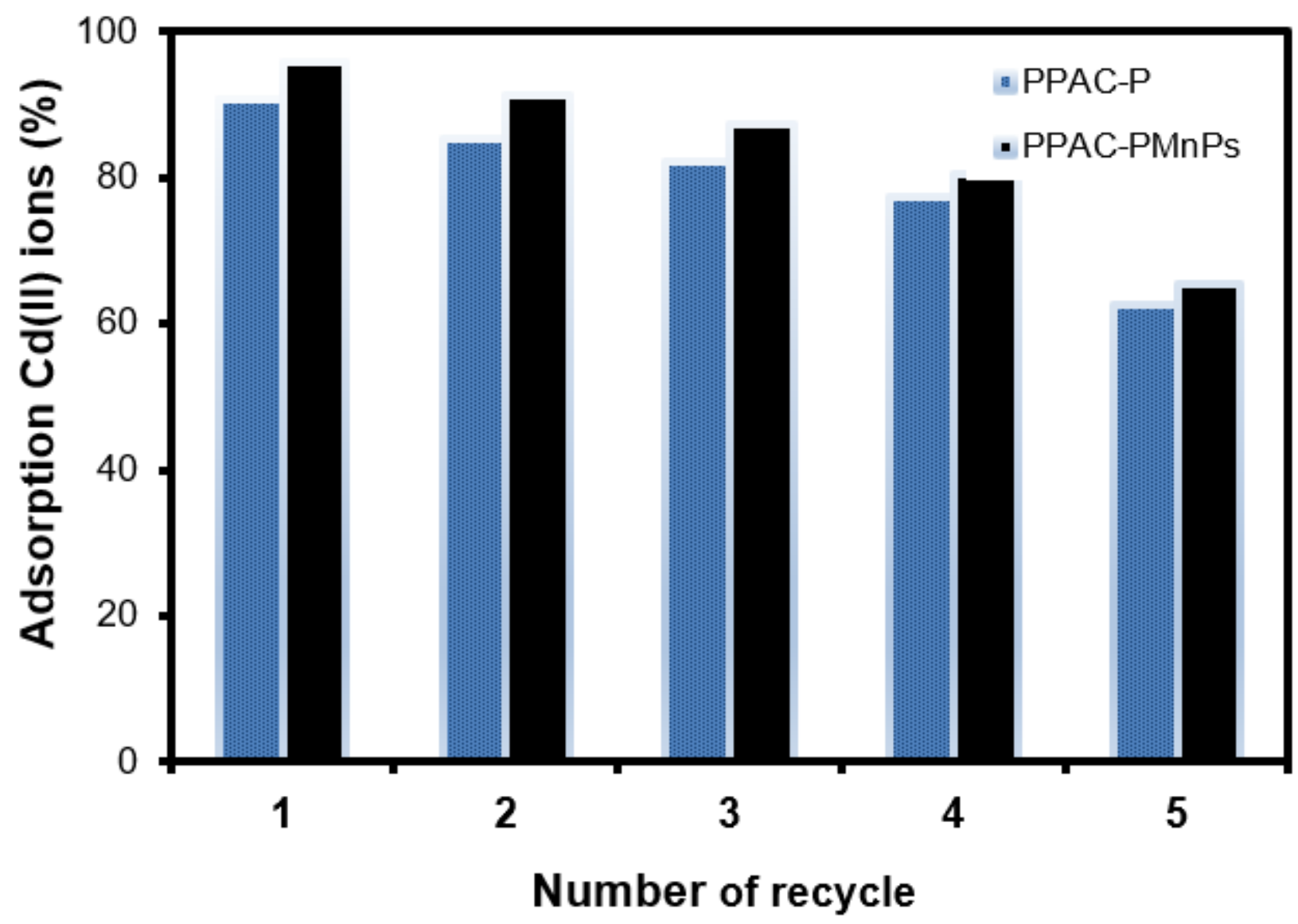

Figure 9

Reusability number efficiency of the adsorption-desorption process of $\mathrm{Cd}(\mathrm{II})$ ion on PPAC-P and PPACMnPs. 